Mirai. Estudios Japoneses

ISSN-e: 2531-145X

http://dx.doi.org/10.5209/mira.67527

\title{
El papel de la tauroflamencología en el universo mishimiano: España y su tradición cultural como ejemplo de resistencia a la homogeneidad occidental
}

\author{
Dámaso Ferreiro Posse ${ }^{1}$
}

Resumen. De entre los numerosos países que contribuyeron a la creación tan única del universo de Mishima, España es uno de los que, a pesar de la impronta que dejó en el escritor, menos investigación existe. La relación de Mishima con nuestro país está llena de altibajos, sinsabores y frustraciones. Sin embargo, y a pesar de ello, el tema de lo español mantiene una presencia inamovible a lo largo de su vida, desde su primera visita a Madrid en 1958 hasta el año de su fatal suicidio en 1970. En el presente trabajo analizaremos de forma pormenorizada el papel de lo español en Mishima, representado básicamente bajo la apariencia del flamenco y la tauromaquia, para, con posterioridad, ver la dimensión cultural que el escritor le confiere en relación con lo japonés y lo occidental. Para ello recurriremos a la presentación de diversos textos en traducción, tanto escritos del propio Mishima como entrevistas realizadas al escritor y allegados, todo ello hilvanado a través de tres importantes figuras que, en sus respectivos campos, condicionaron y forjaron el conocimiento que Mishima obtuvo de España, a saber, Antonio el Bailarín, Katori Kiyoko y Luis Díez del Corral.

Palabras Clave: Mishima Yukio; España; flamenco; tauromaquia; especificidad cultural; alienación cultural

\begin{abstract}
Among the several countries which contributed to the creation of Mishima's universe, Spain has long been forgotten despite the big impression Spanish culture left on the Japanese writer. The relation between Mishima and Spain is full of emotional ups and slumps, deception and frustration. However, Spanish culture is always visible in his writings in one way or another, from his first visit to Madrid in 1958 until his suicide in 1970. This paper aims to clarify the role Spain and its culture, usually under the shape of flamenco and bullfighting, have in Mishima's thought, as well as the cultural dimension the writer gives to Spain in contrast to Japan and some other Western countries. In order to carry out a successful analysis, several texts collected from novels, interviews and newspapers translated into Spanish will be introduced. Moreover, those texts will be connected through three important figures of the time who left an undeniable influence on Mishima: the flamenco dancers Antonio el Bailarín and Katori Kiyoko and the historian Luis Díez del Corral. By doing this, we will also add nuance to our understanding of why Mishima understood Spain in the way he did.
\end{abstract}

Keywords: Mishima Yukio; Spain; flamenco; bullfighting; cultural specificity; cultural alienation

Sumario. 1. Introducción; 2. De la actitud estereotipada de una España exótica al descubrimiento del arte flamenco; 3. Lo español como modelo cultural de referencia: Luis Díez del Corral y la otra cara de la "España negra"; 4. Conclusiones.

Cómo citar: Ferreiro Posse, D. El papel de la tauroflamencología en el universo mishimiano: España y su tradición cultural como ejemplo de resistencia a la homogeneidad occidental, en Mirai. Estudios Japoneses, 4, 2020, 119-128.

\section{Introducción}

Mishima Yukio (1925-1970) es, sin lugar a dudas, el primer escritor japonés que logró desatar un auténtico fenómeno de masas en países tales como Francia, Estados Unidos o Inglaterra. En numerosos países occidentales el número de lectores incondicionales del escritor japonés se multiplicaba año tras año de manera vertiginosa, e incluso se llegaron a hacer adaptaciones cinematográficas de alguna de sus obras, como es el caso de Gogo no eikō [午後の曳航, El marino que perdió la gracia del mar] (1963), traducida al inglés como The Sailor Who Fell from Grace with the Sea (1965) y adaptada a la gran pantalla con el mismo título en 1976.

De la misma forma que el mundo se rendía al genio creador del escritor japonés, el propio Mishima acrecentaba, de forma consciente, su fama y su leyenda a través de una presencia constante en diversos medios de comunicación, controvertidas sesiones fotográficas e incluso cameos en películas. Viajero curioso e incansable, visitó numerosos países a lo largo de su vida. Además de los países canónicos de los que bebió la literatura japonesa durante decenios, tales como Francia, Inglaterra o Estados Unidos, otros tan ajenos a ésta como República Dominicana, México, Puerto Rico o Grecia acogieron al escritor japonés en más de una ocasión y dejaron una honda impronta que él mismo trató en diversas crónicas de viaje, novelas e incluso obras de teatro. Y esto no pasó desapercibido a ojos de

Assistant Professor, Hiroshima University; damasoferreiro@gmail.com 
los investigadores. De esta forma, existe una numerosa bibliografía acerca de la influencia de lo francés, lo griego o incluso lo latinoamericano en la literatura de Mishima. Sin embargo, no todos los países visitados y, por qué no decirlo, admirados por el escritor japonés han sido tratados y estudiados de la misma forma. Un claro ejemplo es el caso de España, que ha sido una de esas grandes olvidadas. En el presente trabajo nos proponemos analizar de la forma más pormenorizada posible el tratamiento que Mishima dispensa a nuestro país. Para ello hemos llevado a cabo un laborioso proceso de selección de aquellos textos y entrevistas en los que el escritor japonés trata la temática de lo español (habitualmente a través de las artes del flamenco y el toreo) a lo largo de su trayectoria profesional. Además, ahondaremos en la evolución que lo español como concepto experimenta en el pensamiento de Mishima, tomando como referencia su encuentro con tres personajes decisivos, a saber, Antonio el Bailarín (Antonio Ruiz Soler, 1921-1996), la bailaora Katori Kiyoko (1920-2009) y el jurista, escritor y politólogo Luis Díez del Corral (1911-1998).

Para llegar a una comprensión más profunda del papel que jugaron el toreo y, sobre todo, el flamenco en el universo mishimiano se han manejado fuentes directas, a saber, los textos traducidos escritos por el propio Mishima; y fuentes indirectas, esto es, las entrevistas a diversos personajes del entorno de Mishima recogidas en las obras de Itasaka y Katori. El hecho de que las obras de Itasaka y Katori no sean de carácter académico, no quiere decir que las entrevistas recogidas en las mismas carezcan de rigor, exactitud y veracidad. Otra cosa son las conclusiones a las que tanto Itasaka como Katori puedan llegar, conclusiones que no han sido recogidas en el presente trabajo.

Antes de pasar al análisis pormenorizado de la cuestión, es necesario hacer una aclaración metodológica importante. En el presente trabajo, pese a que se observa una cierta diferenciación temática que podría conllevar una aparente tripartición de las diversas fases, hemos decidido tratar el flamenco y la tauromaquia como un continuum o evolución natural, y no como una ruptura o cambio de paradigma, debido a las implicaciones históricas y ontológicas que ambas artes comparten.

En cuanto a las mencionadas implicaciones históricas, tal y como Javier Noya ${ }^{2}$ y Alberto Ibáñez ${ }^{3}$ señalan en sus respectivas obras, la imagen de España cambia radicalmente en el siglo XVIII. Debido a las diversas campañas de propaganda motivadas por diversos competidores coloniales extranjeros ${ }^{4}$, España pasa de potencia colonial dominante a país exótico orientalizante y atrasado en un lapso de tiempo relativamente corto. Y con el cambio de la imagen prototípica del país, se produce también una importante modificación de los lugares más representativos del mismo. Si hasta el siglo XVIII la austera imagen de España se identificaba básicamente con Toledo y El Escorial, a partir del XVIII y sobre todo desde el XIX, el epicentro de la imaginería extranjera pasa a ser la pasional Andalucía, sobre todo las ciudades de Sevilla y Granada. Y si hasta el siglo XVIII las labores del país eran básicamente la conquista y la fe, a partir del XVIII y a raíz de la invasión napoleónica, el menester nacional, a ojos de extranjeros, pasa a ser el hedonismo, el cante y baile flamencos y las corridas de toros. No es necesario mencionar que esta imagen adulterada llega a nuestros días potenciada incluso por los propios españoles 5 , y que la imagen que Mishima guarda de España, al menos en un primer momento, no es radicalmente diferente a la que otros países europeos podrían tener.

Sin embargo, es necesario aclarar que, al margen de las motivaciones históricas de carácter peyorativo que se puedan aducir a la hora de tratar el flamenco y la tauromaquia de forma conjunta, desde el punto de vista artístico y formal existe una profunda conexión entre ambas artes que es necesario tener presente a la hora de analizar el tema en cuestión. De esta forma, Manuel Ruíz ${ }^{6}$ habla de tauroflamencología, esto es, disciplina que estudia las semejanzas entre el flamenco y la tauromaquia desde un punto de vista científico y formal:

[Por tauroflamencología se entiende la disciplina que estudia] el conjunto de similitudes estéticas y de talante humano, entre el arte del toreo y el arte flamenco. Unas similitudes que tienen su más aparente reflejo en las semejanzas del vestido o traje de luces de los toreros y la típica indumentaria de los artistas flamencos, principalmente la de los bailaores, así como la similitud de los desplantes y los juegos de brazos, quiebros de cintura y otras actitudes, que caracterizan algunos momentos de la interpretación de ambas artes. Mas la analogía más profunda entre toreo y flamenco radica (...) en el sentido afín de concebir, sentir y realizar el arte por los artífices de estas dos artes españolas, poniendo de manifiesto motivaciones anímicas muy comunes ${ }^{7}$.

De esta forma y como hemos señalado, el tratar el tema del flamenco y la tauromaquia, o lo que es lo mismo, la tauroflamencología en la obra de Mishima, obedece a criterios metodológicos específicos. Y es que, si bien es cierto que en los textos del escritor japonés aparece una cierta división entre la etapa del flamenco (en los primeros años) y de la tauromaquia (en los últimos), ambas disciplinas estás formalmente ligadas y comparten una serie de características intrínsecas que permiten un análisis conjunto. Como Manuel Ríos Ruiz muy bien señala:

Noya, J. (2012).

Ibáñez, A. (2018).

Íbidem: 67-79.

Alberto Ibáñez utiliza el término hispanobobos para referirse a todos los españoles que potencian de forma banal y sin conocimiento de causa los estereotipos del país.

Ríos Ruiz, M. (2019).

Íbidem: $65-66$. 
[...] tanto el toreo como el cante y el baile tienen en la espontaneidad su expresión más verídica y artísticamente más valiosa, son artes que requieren de sus intérpretes unas cualidades especialísimas, cifradas primordialmente en una sutil y a la par profunda sensibilidad, gracia, sentimiento trágico y donosura, todo un compendio de racial temperamento ${ }^{8}$.

\section{De la actitud estereotipada de una España exótica al descubrimiento del arte flamenco}

Mishima realizó dos viajes a España a lo largo de su vida, uno en 1958 y otro en 1960. El primero de ellos tuvo una motivación un tanto peculiar. Tras el éxito de la publicación de sus obras de teatro Kindai Nōgaku-shū [近代能楽集, Compilación de obras contemporáneas de teatro nô] el escritor japonés viajó a Estados Unidos, concretamente a la ciudad de Nueva York, para supervisar de primera mano la puesta en escena y asistir, de paso, a su estreno internacional. Sin embargo, debido a diversos problemas, el proyecto no salió adelante y la puesta en escena se canceló, lo que provocó una profunda decepción en Mishima. Como forma de pasar página y recomponerse, el escritor decidió llevar a cabo un viaje en el que visitaría dos capitales europeas, a saber, Madrid y Roma, antes de volver a Japón.

A raíz de esta primera visita a la capital española, Mishima escribió y publicó dos breves textos, uno en 1958, Furamenko no shiroi mosuso [フラメンコの白い裳裾, Los volantes blancos del baile flamenco], incluido en su crónica de viajes titulada Tabi no ehon [旅の絵本, Libro ilustrado de mis viajes] y otro en 1960 titulado Madoriddo no Ōmisoka [マドリッドの大晦日, La Noche Vieja madrileña], incluido en el libro de Inoue Munekazu titulado Yōroppa no machikado de [ $コ$ ロッパの街角で, Por las calles de Europa]. Lo que llama la atención de Mishima en este primer contacto con lo español son básicamente dos elementos que habitualmente sorprenden a todo extranjero que visita España: la belleza del baile flamenco y la diferencia cultural existente entre España y los demás países europeos del entorno. Con respecto al flamenco, Mishima relata su visita al tablao madrileño Zambra de la siguiente forma:

España, Madrid, Tablao Zambra. En este local pude apreciar desde muy cerca y con claridad tanto los movimientos de los brazos como el taconeo, así que realmente disfruté el espectáculo. (...) Para mí, el momento más hermoso fue cuando la bailarina, izando sus brazos dio una palmada y, acto seguido, chasqueando sus dedos arqueó su pecho, y con un taconeo sonoro se fue acercando hacia los espectadores. Se quedó allí, al borde del escenario y, girando su cuerpo lentamente, se fue poniendo de espaldas. Ese fue el momento. (...) La bailaora bullía y se veía arrastrada sobre el escenario (...) como si de un torrente que se retuerce se tratase, su espalda en tensión sostenía una hermosa cerviz y mientras tocaba las palmas atravesaba el aire con sus manos como si fuesen afiladas cuchillas. Su cuerpo, traspasado por la cadencia del taconeo, grácil y vigoroso a la vez, se iba alejando de nosotros hacia el fondo del escenario. (...) Y lo mismo hacía la música aunque, como es lógico, ésta no dejaba ver su espalda. La bailaora, mostrando sus largos y blancos volantes, lo hacía de una forma más certera, y más hábil que la sombra de un pájaro que se distancia al vuelo. Y así, al compás de su taconeo, ella iba apoderándose de mi tiempo, un viajero cualquiera que, como yo, se encontraba en un rincón de Madrid9.

El tablao que Mishima visita en 1958, de nombre Zambra, es un local del céntrico barrio de los Jerónimos de Madrid, en activo desde el año 1954 hasta el año 1975, año en que fallece su fundador Fernán Casares. Este tablao, uno de los mayores exponentes del auge que experimenta lo flamenco en la capital española durante los años cincuenta y sesenta (en buena parte debido al aumento de turismo), se convierte rápidamente en el principal referente del baile flamenco, el "baluarte de pureza" de la capital ${ }^{10}$, tanto por la estrategia publicitaria que sigue su fundador como por la gran calidad de su artista principal, Rosa Durán, a la que muy probablemente Mishima vio sobre el escenario.

Además del texto previamente traducido, Mishima también incluyó en la portada de su crónica de viajes una representación del traje español con peineta y mantilla y una escena de tauromaquia al fondo en la que aparece representado un picador a caballo, todo ello a color y compartiendo espacio con fotografías del desierto norteamericano, modernas urbes con rascacielos y danzas de nativos sudamericanos con tocados de plumas de vivos colores. En el interior del libro, diversas fotografías de su visita a Toledo y a El Escorial se entremezclan con fotografías de espectáculos de ballet, musicales y obras teatrales de Estados Unidos y la URSS.

En el segundo texto acerca de esta primera visita a España, "La Nochevieja madrileña”, Mishima incide en un aspecto fundamental, a saber, las diferencias y semejanzas de España frente a otros países europeos. En dicho texto se puede leer lo siguiente:

El invierno madrileño es bastante frío. En este país, menospreciado por ser el África de Europa, sólo el frío es propiamente europeo. (...) En medio de este panorama de llegada del Año Nuevo, a excepción del clima, todo es

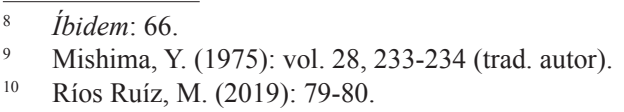


propio de un país sureño, un país en el que las negras pupilas de las mujeres que asoman entre las sombras de sus mantones hacen olvidar este frío por completo ${ }^{11}$.

Estos dos primeros textos se adaptan perfectamente al propósito de las obras en las que aparecen recogidos. Como crónicas de viajes que son, tratan básicamente lo diferente, lo que llama la atención del viajero. Como hemos señalado anteriormente, plantean España desde el prisma de lo exótico y lo incomprensible. Al no existir un desarrollo de la acción concreto e interconectado, el escritor apela a una técnica muy básica que es la mera descripción de hechos y paisajes desde una óptica personal. Sobra decir que esta búsqueda del exotismo no es exclusiva de Japón ni mucho menos. Sin embargo, y sobre todo a partir de la Restauración Meiji, este ikoku jōchō ${ }^{12}$, como se designa en japonés, juega un papel fundamental en literatura y acaba por convertirse en la obsesión de numerosos intelectuales y escritores de finales del siglo XIX y principios del XX, aunque si bien es cierto, ese sentimiento de búsqueda de lo exótico tiende a relacionarse con la Europa canónica más que con la periférica. Esta primera aproximación a España por parte de Mishima se caracteriza pues por su superficialidad, así como por recoger de forma clara y concisa los estereotipos que existen del país. Dicho de otra forma, Mishima plasma en sus textos lo que se supone que debe aparecer en cualquier publicación sobre España.

Tras esta primera visita a nuestro país, Mishima realiza una segunda, de nuevo a Madrid y alrededores, como parte de su larga luna de miel en 1960, esta vez acompañado por su esposa Yōko. También en esta ocasión Mishima acude a un tablao flamenco. Sin embargo, pese a haber viajado a España acompañado de su mujer, decide ir solo, dejándola a ella en la habitación del hotel. Mishima no escribe sobre esta segunda visita, pero las palabras de su esposa atestiguando el hecho están recogidas en la obra de Itasaka Gō ${ }^{13}$ :

"Hasta aquel momento nunca había pasado nada igual, pero aquel día, de repente, me dejó completamente sola en el hotel y se fue. A las cinco horas y pico volvió y le pregunté a dónde había ido. Me respondió que a un espectáculo de flamenco. Yo estaba un poco enfadada así que medio gritando le dije que por qué no me había llevado con él. Entonces, en ese momento, con semblante abatido, me respondió diciendo que él pensaba que yo me aburriría. No sé, fue todo muy raro"14.

El interés de Mishima por el flamenco va haciéndose más y más intenso con el paso del tiempo, tanto que en diversas ocasiones busca la forma de empezar a aprenderlo. Y es a través de una mayor comprensión de este arte que la imagen que Mishima tiene sobre España comienza a cambiar y a acercarse a su universo creativo e ideológico. En su encuentro con el flamenco destacan dos personalidades que le dejaron una profunda impronta, a saber, el sevillano Antonio Ruiz Soler y la japonesa Katori Kiyoko. El primero de ellos, popularmente conocido como Antonio el Bailarín o el Gran Antonio, fue uno de los máximos exponentes del flamenco a nivel nacional e internacional durante décadas. El bailaor sevillano, tras separarse de su histórica pareja de baile Rosario en los cincuenta, además de su faceta sobre las tablas, comienza a trabajar también como coreógrafo y director artístico llevando su compañía de danza, Antonio Ballet Español, por numerosas ciudades del mundo. Entre gira y gira, Antonio realiza largas estancias en Madrid y Barcelona y, en la década de los sesenta, también actúa en tablaos flamencos de la capital española como artista invitado, sobre todo en el Tablao Zambra ${ }^{15}$, al que había acudido Mishima en su primera visita a España. No se tiene registro de si en la segunda visita de Mishima a España, Ruiz Soler actuó en un teatro o un tablao, o de si Mishima vio o no en vivo al sevillano, por lo que aventurar que lo conociese en persona es un tanto arriesgado. Itasaka, en una entrevista que realizó a Ogawa Satoshi, gran conocedor y amigo de Mishima, recoge las siguientes palabras:

Cuando visité la casa de Mishima, había un montón de cosas españolas que saltaban a la vista ${ }^{16}$, y yo, como de eso no entiendo mucho, pensé que era todo de Grecia. Me acuerdo perfectamente porque fue algo con lo que no contaba. Y entonces, un día, cuando estaba hablando con él por teléfono, le pregunté si le gustaba España. Me dijo que sí, pero un sí un poco seco, como sin querer entrar en el asunto. Yo lo interpreté como le gustaba sin más. Sin embargo, de repente empezó a decir que le gustaba un bailaor español. (...) Mishima nunca me reveló el nombre de ese bailaor. Creo que fue Isoda Kōichi quien, años después, me lo comentón ${ }^{17}$

Isoda Kōichi fue un crítico literario y especialista en literatura inglesa que trabajó, fundamentalmente, en el campo de la estética y la representación del mal, el sufrimiento y el martirio cristiano, temas sobre los que giran sus obras más representativas ${ }^{18}$. Viendo los temas que Isoda trata, es más que fácil imaginar la posible afinidad que

\footnotetext{
Mishima, Y. (1975): vol. 30, 16 (trad. autor).

En japonés 「異国情調」。

13 Itasaka recoge en su obra numerosas entrevistas realizadas a Yōko, esposa de Mishima, así como a diversos amigos y conocidos del escritor. Dichas entrevistas se recogen el presente trabajo por su valor documental. 
hubiese podido existir entre Mishima y él. Sin embargo, algo importante que también apunta Itasaka es que no está muy claro si fue Isoda quien le habló a Mishima de Antonio el Bailarín o si, por el contrario, fue el escritor quien lo descubrió por su cuenta (puede que incluso en Madrid) y se lo comentó a Isoda después. Sea como fuere, el hecho de que tanto Mishima como Isoda se hubiesen fijado en el bailaor tampoco es algo excepcional. Principal referente del baile español en el extranjero tras la conquista del público americano, Antonio Ruiz alcanzó una fama a nivel internacional sin parangón, y con su compañía de baile visitó Japón en diversas ocasiones (bien conocida es, por ejemplo, su última actuación en Hokkaido antes de retirarse definitivamente del mundo de la danza). Pero Antonio Ruiz no era conocido únicamente por su destreza como bailaor. Su ajetreada vida sentimental con personajes de primer nivel así como su condición sexual eran secretos a voces ampliamente comentados ${ }^{19}$. Y de la misma forma que corrieron ríos de tinta acerca de su vida personal, lo mismo ocurrió con el estilo de baile que desarrolló. Antonio fue muy criticado en ciertos círculos conservadores y puristas tanto por desarrollar un tipo de baile de rasgos femeninos como por vestir colores vivos y atrevidos, como el rojo, tradicionalmente reservados a las mujeres ${ }^{20}$. El caso de Ruiz Soler no es algo totalmente extraordinario, pues, si bien es cierto que el mundo del flamenco y la copla se caracteriza por una clara diferenciación entre lo masculino y lo femenino ${ }^{21}$, también es cierto que nunca han faltado intérpretes que se atreviesen, de una forma u otra, a trasgredir y poner en solfa tal compartimentación. Antonio Mairena, Miguel de Molina, Juanita Reina o Pedrito Rico son sólo algunos ejemplos.

Como hemos señalado hasta el momento, en la obra de Mishima no hay registros del nombre de Antonio Ruiz Soler y, de no ser por las menciones de sus más allegados, sería imposible llegar a tal conclusión. Sin embargo, es de reseñar que la existencia de un "misterioso" bailaor flamenco tiene una presencia más que patente en la imaginería del escritor japonés. El texto anterior en el que Ogawa y Mishima conversan continúa de la siguiente forma. Esta vez es Mishima el que habla: “¿No te parece que si hubiera un hombre bailando flamenco en este patio ${ }^{22}$ bajo la luz de la luna llena sería igual de hermoso que una pintura? De no existir las novelas, yo creo que habría sido bailaor. Más bien, me gustaría haber sido bailaor."23

Este breve texto, con el "me gustaría haber sido..." como colofón, recuerda sobremanera a la obra con la que Mishima se da a conocer como escritor, Confesiones de una máscara. En el primer capítulo, cuando el protagonista relata los recuerdos de su infancia, menciona cómo le gustaría ser de mayor un muchacho que se dedica a recoger inmundicias, revisor de tren, conductor de tranvía, soldado,... oficios todos ellos que tienen en común tres motivos fundamentales, a saber, un uniforme con pantalones más o menos ajustados, una referencia odorífera destacable (gasolina, sudor, etc.) y, sobre todo, una fuerte impresión de un vivir marcado por la tragedia. Esta forma de entremezclar el dolor existencial con la pulsión sexual o el erotismo ${ }^{24}$ coincide claramente con la visión que Mishima tiene del flamenco bailado por hombres. Los pantalones ajustados de los bailaores, el sudor y, sobre todo, la conexión del pueblo gitano, condenado a la marginalidad y el ostracismo, con el sentir trágico encajan perfectamente en la estética de Mishima. Sin embargo, la obsesión del escritor japonés por el flamenco va todavía más allá y es tan intensa que, tras su segunda visita a España, comienza a frecuentar los tablaos de Tokio, en soledad o en compañía de amigos. Es en esta época cuando conoce a Katori Kiyoko, una de las más famosas bailaoras del momento en Japón. En las memorias que ésta publica al cumplir los 85 años ${ }^{25}$, Katori relata su primer encuentro con Mishima:

"Me han pasado cosas curiosas con diversas personas. En 1963, justo al volver de España, tuve una actuación en el Sankei Hall y Mishima Yukio vino a verme. No es que viniese a verme a propósito, sino que parece ser que fue más bien una casualidad. Cuando acabé de bailar y me fui de vuelta al camerino me avisaron de que había alguien que quería verme, y ¡cuál fue mi sorpresa al saber que no era otro que Mishima! El Mishima de aquel entonces no es que me agradase demasiado, pues lo asociaba a fotos y anuncios publicitarios un poco raros. Sin embargo, el Mishima que vino a verme, sin mediación de nadie y en compañía del artista Okamoto Tarō, estaba muy lejos de esa imagen que proyectaba al mundo, pues fue muy cordial y educado conmigo. Me dijo que quería aprender a bailar flamenco. Lo cierto es que también su esposa estaba loca por el flamenco, y ésta le había prometido que, tras aprender ella, sería ella la que le enseñaría a él."26

Itasaka le preguntó a Yōko, esposa de Mishima, la razón por la cual no quería que su esposo acudiese a las clases de Katori. El motivo de la reticencia de ésta se debía a que, al parecer, Mishima no tenía sentido del ritmo ni era muy

19 Arriazu, S. (2006): 202.

20 Fernando López (2017) va un paso más allá y habla no de lo femenino en Ruiz Soler sino de una "masculinización indirecta de (su) danza para desactivar la rareza, lo queer que hay en ella, a través del virtuosismo en zapateados, giros y saltos. (...) Con independencia de ciertos elementos que en su danza podrían hacer "sospechoso" a Antonio, el Bailarín desarrolla una estrategia de autoprotección mostrándose como alguien con una gran capacidad performativa, lo que permite al espectador hombre heterosexual mirarle con cierta admiración por ser capaz de hacer lo que hace," p. 89.

21 Baste para ello la diferenciación entre "bailar en hombre" y "bailar en hembra", recogida en el Decálogo para bailaores de Vicente Escudero de 1951. Escudero Urive, V. (2017).

22 Hemos optado por traducir la palabra nakaniwa como patio. Sobra decir que las casas japonesas no cuentan con un patio semejante al de las casas españolas. La traducción literal sería "jardín interior".

23 Itasaka, G. (1997): 31. (trad. autor)

24 Sobre la relación entre violencia y erotismo en el pensamiento de Mishima se aconseja consultar Mishima, Y. (2015): 36-38.

25 La obra en cuestión es Katori, K. (1998).

26 Katori, K. (1998): 114. 
habilidoso en lo que a coordinación corporal se refiere. En otras palabras, no quería que su marido acabase haciendo el ridículo en público. Tras este episodio, fue Yōko quien comenzó a acudir al estudio de Katori para aprender ${ }^{27}$.

Tras esto, Katori cuenta otro suceso con Mishima de protagonista todavía más interesante si cabe. Tras haberse negado a enseñarle a bailar por imperativo de su esposa, Mishima acudió de nuevo a Katori y pidió a la bailaora que lo ayudase a escribir obras de teatro de inspiración flamenca, es decir, obras de teatro en las que el baile y el cante ocupasen un lugar destacado. El entusiasmo que el escritor desprende ante tamaña empresa se transluce a través de las siguientes palabras: "Voy a escribir obras de teatro flamencas que dejen a los españoles boquiabiertos" ${ }^{28}$. Katori relata este episodio de la siguiente forma:

"Lo cierto es que Mishima me dijo en numerosas ocasiones que quería escribir obras teatrales de inspiración flamenca y que quería que yo bailase en ellas. Me lo pedía con un entusiasmo increíble, hasta me lo suplicaba. Guiones escritos por él. Además, decía que ya buscaría la forma de poder sufragar los gastos que se pudiesen originar. (...) Sin embargo, yo no tenía la confianza suficiente en mí misma como para poder hacerlo. Y mi marido también se opuso diciéndome que qué haría en caso de hacer fracasar a un escritor de la talla de Mishima. Por eso, al final rechacé su propuesta." ${ }^{29}$

A través de los textos, entrevistas y testimonios anteriores hemos podido demostrar de forma fehaciente que, pese a la escasez de escritos relacionados con el tema, el interés de Mishima por el flamenco es más que patente. Si bien es cierto, dicho interés no dio los frutos que el escritor posiblemente esperaba. $\mathrm{Y}$ tras la imposibilidad de aprender a bailar y al no contar con la ayuda deseada para escribir las obras que le habría gustado, Mishima tira la toalla y cambia el flamenco por en kendo y la musculación, entrando en su bien conocida fase de culturista. Ahora bien, es importante destacar que el interés de Mishima por lo español no se limita al flamenco, sino que más bien, el flamenco y su experiencia frustrada representan la puerta de entrada a un mundo por el que acabará sintiendo una afinidad inesperada.

\section{Lo español como modelo cultural de referencia: Luis Díez del Corral y la otra cara de la "España negra"}

Tras el interés que despierta en Mishima el arte del flamenco, el escritor japonés comienza a adquirir una base más teórica acerca de la cultura e historia españolas mediada por la figura del escritor Luis Díez del Corral. La relación entre Díez del Corral y Mishima está fielmente documentada en una de las obras sobre el escritor japonés que mayor impacto ha causado en España: Mishima o el placer de morir, escrita por el psiquiatra y escritor Juan Antonio Vallejo-Nájera. Teniendo como objetivo último el desentrañar la causa psicológica que conduce a Mishima a cometer un suicidio tan sangriento, Vallejo-Nájera lleva a cabo un exhaustivo proceso de documentación inédito hasta el momento, viajando a Japón e incluso entrevistándose con la viuda del escritor. Si bien es cierto que Vallejo-Nájera no contaba con conocimientos sobre lengua japonesa, lo cual limita en cierta manera el análisis del tema en cuestión, es necesario reconocer que el esfuerzo realizado y la calidad del trabajo resultante son más que loables.

En el presente trabajo, nos limitaremos a una mera presentación de la relación entre Mishima y Díez del Corral para centrarnos, sobre todo, en el análisis de varios textos literarios en los que se trata la temática de lo español ${ }^{30}$. Díez del Corral, tras publicar diversas obras y artículos entre los que destaca El rapto de Europa. Una interpretación histórica de nuestro tiempo ${ }^{31}$, es invitado por Ortega y Gasset a ir en su nombre a diversas charlas organizadas por varias universidades japonesas en $1962 \mathrm{y}$, tras su gran acogida, volverá con posterioridad en $1968^{32}$. Es en esta primera visita cuando el político e historiador español se encuentra por primera vez con Mishima, que asiste a la primera de estas clases en la Universidad de Tokio. Fascinado por conceptos como el buen morir o el código de honor calderoniano, el escritor japonés invita a Díez del Corral a su casa en numerosas ocasiones y, con traductor primero y sin él después, hablan largas horas acerca de todo ello. Parte de lo que ambos hablaron en estas jornadas está recogido en la breve entrevista Higashi to Nishi, publicada primero en japonés por la editorial Miraisha en $1969^{33}$ y traducida con posterioridad al español bajo en nombre Oriente y Occidente ${ }^{34}$.

Lo que llama la atención de Mishima en su contacto con la cultura española es algo que él creía exclusivo de Japón, esto es, la búsqueda de la belleza y el honor en la muerte. Conceptos como el buen morir, en desuso en la España actual, se pueden englobar en una categoría más amplia que a finales del siglo XIX comienzos del XX se comenzó a denominar, de forma despectiva, la "España negra". Una España oscura en la que las supersticiones más inverosímiles, un código de honor vetusto y un morboso gusto por la sangre y la muerte se oponían a la brillantez y al avance europeo haciendo que España quedase anclada en un pasado nebuloso y al margen del progreso. $\mathrm{O}$ al menos, esa era la idea que Émile Verhaeren y Darío de Regoyos tenían cuando escribieron y publicaron sus cuadernos de la

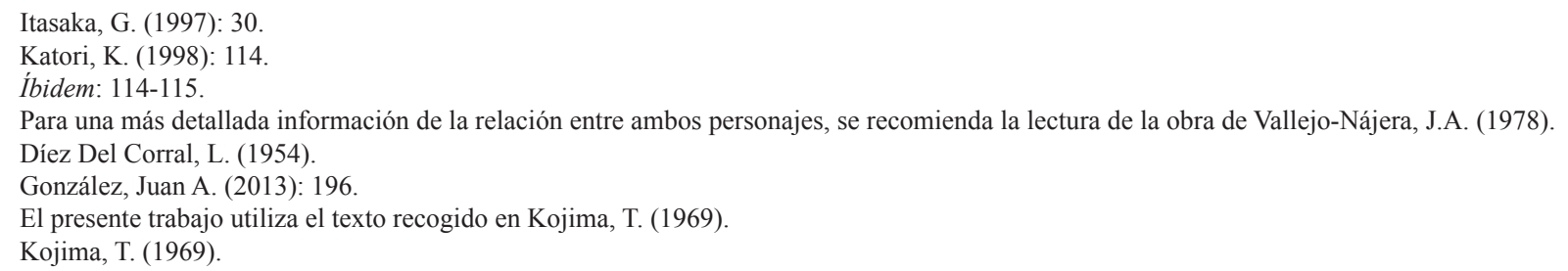


España negra ${ }^{35}$ en 1898. Años después, este concepto tremendista de España es retomado por José Gutiérrez-Solana, pintor y escritor expresionista que en 1920 publica, a imitación del trabajo que habían realizado varios decenios antes Regoyos y Verhaeren, las experiencias resultantes de su viaje por varias capitales en la obra homónima La España negra (1920). Y no sólo escritores, sino también diversos pintores como Isidre Nonell, Ignacio Zuloaga o el propio Gutiérrez-Solana se dedicaron a retratar la cara más oscura del costumbrismo de España. Andrés Trapiello relata lo siguiente en el prólogo de su edición a la obra de Gutiérrez-Solana:

"Solana es (...) radical. Apenas nos habla de valores morales, y sin embargo la conclusión que sacamos de sus pinturas literarias y de sus plásticos relatos es que en España tendría que cambiar todo para que se hiciera respirable, pero que el drama vendría luego, porque si cambiara, dejaría de ser España, y desaparecería. Sin duda, el escritor habría estado de acuerdo con el diagnóstico ganivetiano: "España es una nación absurda y metafísicamente imposible", escribió el romántico Ganivet, "y el absurdo es su nervio y su principal sostén. Su cordura será la señal de su acabamiento.” (...) Solana cree que en el momento en que España deje de ser negra, dejaría de ser España."36

En esta España irracional dibujada por la corriente tremendista, la Fiesta Nacional, esto es, la tauromaquia, ocupa un lugar de excepción. La sangre y los cadáveres desollados y a medio descomponer de las víctimas que las corridas se cobran, principalmente caballos y asnos, son arrojados de cualquier manera por las calles colindantes y las descripciones de tales miserias ocupan páginas y páginas de estas obras que buscan retratar la tendencia necrofílica del país. Esta realidad puede ser entendida de forma positiva o negativa dependiendo del intelectual que a ella se aproxime. Pues si bien la sangre y la muerte forman parte inseparable de la realidad española, éstas no tienen por qué ser entendidas siempre de forma negativa.

A raíz de conocer a Díez del Corral, Mishima entra en contacto con estos conceptos tan ajenos a la racionalidad europea. Dicho de otra forma, Mishima se aproxima a la especificidad española desde un punto de vista teórico y, lo que es más importante, desde una perspectiva aséptica y libre de prejuicios estereotipados impuestos por el exterior. En este proceso, y para sorpresa del propio Mishima, las similitudes que encuentra entre España y Japón son, a sus ojos, muy numerosas, y sobre ellas escribe en diversas ocasiones a lo largo de sus últimos años. Para aproximarnos a la visión del escritor, presentaremos tres breves textos en traducción y uno en inglés que recogen su parecer.

"De entre los diversos trabajos desempeñados por hombres, es difícil encontrar uno que sea más duro, ponga la propia vida en juego y haga de la elegancia y la belleza su esencia. A diferencia de los malolientes y sucios cowboys estadounidenses, los toreros, como si de cortesanos se tratasen, visten elegantes trajes de seda. Debajo de esas coloridas vestimentas no llevan protecciones y, por no llevar, no llevan ni ropa interior, lo cual es muestra de su verdadera determinación. El hecho de que sea adecuado para un hombre vestir prendas tan coloridas y magníficas es única y exclusivamente porque en el toreo todo tiene relación con la muerte, la valentía y el derramamiento de sangre. De hecho, los kimonos ribeteados con tantas florituras de cantantes de moda actuales me parecen absolutamente detestables. En cambio, los toreros resultan hermosos, hermosos gracias al peligro, y todavía más aún cuando los ronda la muerte. Y este es el motivo por el que, para el común de los hombres de a pie, por mucho que les duela, tal belleza les resultará inalcanzable." ${ }^{37}$

En este texto tenemos los elementos básicos de la estética de Mishima, a saber, la muerte, el dolor, la sangre, la belleza y la masculinidad, todos ellos perfectamente representados, según el escritor, en el mundo del toro. Años más tarde, Mishima vuelve a tratar el motivo del toreo en la que es considerada la obra cumbre de sus últimos años, la trilogía de El mar de la fertilidad, en los siguientes términos:

"Haciendo caso omiso de los animalistas del mundo, España, a diferencia de Japón, decidió conservar el toreo como seña de identidad nacional. Y es que Japón, a raíz de la Restauración Meiji, aspiró a deshacerse de todo aquello que podría ser considerado "bárbaro". Como resultado, el alma más pura y auténtica del pueblo japonés se vio obligada a refugiarse en el subsuelo y, erupcionando de cuando en vez, hace gala de un poderío violento al tiempo que se convierte en lo más abominable y temible de cada persona." ${ }^{38}$

Este pequeño fragmento ofrece una clara comparación entre España y Japón, más exactamente del rumbo que ambos países deciden tomar a la hora de defender su patrimonio cultural al entrar en contacto con lo "foráneo". Según Mishima, mientras en Japón se decide lo que es culturalmente aceptable o no según la hipócrita doble moral puritana occidental, España, a ojos del escritor, posee un código propio e independiente que hace que por una parte se convierta en blanco de críticas y burlas por parte de países del entorno pero que, por la otra, no niegue su propio ser. Y es gracias a ello que el país carece de problemas de crisis identitarias que Japón sí debe afrontar de cuando en vez. Según este fragmento, podemos fácilmente intuir que, desde la perspectiva de Mishima, Japón y España tienen un

\footnotetext{
La primera edición de dichos cuadernos aparece recogida en la revista Luz, dirigida por el propio Darío Regoyos. Su publicación en formato de libro acompañando el texto con diversas ilustraciones se produjo un año después, en 1899.

36 Gutiérrez-Solana, J. (2000).

37 Mishima, Y. (1975): vol. 32, 351-352. (Trad. autor).

38 Mishima, Y. (1975): vol. 4, 234. (Trad.autor).
} 
mismo punto de partida, pero los resultados que ambos países alcanzan son muy desiguales en función de si protegen o rechazan lo patrio. Este aspecto será más ampliamente desarrollado en el apartado de las conclusiones.

En el siguiente fragmento, tomado de la publicación anteriormente mencionada de Kojima en la que conversan Mishima y Díez del Corral, el escritor japonés habla de la cultura española desde una perspectiva más amplia yendo más allá del cliché de los toros y el flamenco:

"MISHIMA: (...) Lo que más admiro de la cultura española es el orgullo y la valentía, algo que brilla por su ausencia en los japoneses de hoy día. Cuando hablo con españoles, siempre siento como que quiero recuperar esos sentimientos de orgullo y valentía que antiguamente los buenos japoneses poseían. Cuando los extranjeros hablan del carácter de los españoles, suelen simplificar diciendo que tienen unos sentimientos muy exacerbados o que son un pueblo muy orgulloso. Eso, pese a lo petulante que pueda parecer, es lo que se solía decir antiguamente de los buenos japoneses. Además, también sentí en numerosas ocasiones la similitud que existe entre el arte popular español y el japonés. El mejor ejemplo es el caso del teatro de García Lorca. Y es que el que sus obras nos lleguen tan fácilmente al corazón es porque los japoneses somos capaces de comprender esa alma popular española. Otro pequeño ejemplo es el de la sensibilidad que desprenden los intervalos del flamenco, y es que no conozco otro baile europeo que se le parezca. Dichos intervalos me resultan muy semejantes a los del teatro nō, o a los del kabuki y, sobre todo, a la cadencia del bunraku. Más aún, la canción española ${ }^{39}$ tiene un sentimiento de tristeza muy profundo que se asemeja en su esencia al que existe en la música folclórica japonesa. Quién sabe, todo esto podría deberse a la influencia oriental que existe en España." ${ }^{20}$

Para finalizar, aportaremos un último texto, esta vez en inglés ${ }^{41}$, que el escritor japonés publicó en el periódico británico The Times el veinticuatro de septiembre de 1969. En este artículo, titulado "A problem of culture", el escritor japonés expone algunos de los posicionamientos de su famoso ensayo Bunka Bōeiron (1969) para, al final, hacer una asimilación entre la cultura japonesa y la española a raíz de la rebelión de samuráis llevada a cabo el veinticuatro de octubre de 1877 en Kumamoto, conocida como la Rebelión Shinpüren:

"In the first 20 years of my life national culture was controlled by the unnatural puritanism of the militarists. For the past 20 years pacifism has been sitting heavily on the samurai spirit, a burden on the easily stimulated Spanish soul of the Japanese. The hypocrisy of the authorities has permeated the minds of the people, who can find no way out. Wherever national culture seeks to regain is totality, almost insane incidents occur. Such phenomena are interpreted as the undercurrent of Japanese nationalism, intermittently bursting out like lava through cracks in a volcano." ${ }^{42}$

La base de este artículo es el incidente que tiene lugar en agosto de 1969 en el antiguo aeropuerto de Tokio, según el cual un joven japonés intenta herir al Secretario de Estado Norteamericano William Rogers con un objeto punzante. La reacción de condena histérica que hacen los medios de comunicación japoneses del momento llama la atención de Mishima que, sin justificar en ningún momento dicho ataque, intenta aportar su visión de lo ocurrido. El motivo principal, como Mishima recoge en su texto, no es otro que el de la venganza: soldados norteamericanos hirieron con sus bayonetas a activistas japoneses que protestaban por las bases americanas en Okinawa y, a su vez, el joven japonés se vio impelido a proporcionar un daño similar al "enemigo". Sin embargo, según Mishima, esta histeria colectiva desatada a raíz del ataque no es más que una muestra de lo artificial que resulta la postura de paz absoluta adoptada por Japón tras la Restauración Meiji. Este episodio lo compara luego con el asesinato del dirigente comunista Asanuma Inejirō a manos de Yamaguchi Otoya, asesinato tras el cual el joven de diecisiete años decide quitarse la vida. Y sigue a esta comparación la anteriormente mencionada Rebelión Shinpüren, en la que todos los samuráis que sobrevivieron al ataque de armas occidentales decidieron quitarse la vida siguiendo el ritual del seppuku. En otras palabras, estos conatos de insubordinación o incluso rebelión son claros ejemplos de lo que Mishima denomina "erupciones" inevitables del "carácter japonés", y cuya causa reside en haber sacrificado la tradición propia en favor de la modernidad occidental. El ser japonés, muy próximo al ser español ("Spanish soul of the Japanese"), se ve incapaz de responder a los diversos desafíos culturales que el devenir de la historia plantea debido a la castración cultural sufrida, se muestra al mundo con su más amable rostro mientras permanece atado de pies y manos. Sin embargo, el reverso de la moneda existe y, de cuando en vez, "el orgullo y el valor" emergen como si de la lava de un volcán se tratasen, poniendo de manifiesto una realidad lejos de la apariencia adoptada de cara al exterior.

\section{Conclusiones}

A partir del análisis de los textos anteriores, sobre todos los cuatro últimos, podemos sacar una serie de conclusiones más o menos evidentes que pueden dar cuenta de la idea que Mishima tiene de España, así como de la relación que,

\footnotetext{
Mishima utiliza el término minyō, es decir, canción folclórica. El término canción folclórica en España goza de una riqueza extraordinaria y está compuesto por diversos géneros musicales (copla, zambra, pasodoble, etc.), habitualmente englobados bajo la nomenclatura de canción española.

40 Kojima, T. (1969): 189-190.

41 El texto original fue escrito en inglés.

42 Mishima, Y. (1969): IX.
} 
a su parecer, la cultura española puede tener con la japonesa en un nivel más profundo y lejos de lo meramente superficial. Lo primero que cabe reseñar es que, como hemos mencionado al comienzo del presente trabajo, la imagen que Mishima tiene de nuestro país evoluciona con los años. La profundidad y el contenido de los textos de la primera etapa, que no son más que meras descripciones del espectáculo flamenco o del panorama de la Nochevieja madrileña, no tienen nada que ver con los textos de sus últimos años sobre el toreo, en los que lo español aparece claramente vinculado a los típicos elementos de la estética mishimiana, lo cual denota un conocimiento sobre nuestro país lo suficientemente profundo como para poder compararlo con la realidad del suyo propio. El flamenco del tablao Zambra, que a ojos del joven Mishima, no deja de ser un exotismo ameno y divertido de un "país sureño", se convierte en una obsesión a raíz de su segunda visita a España, y ello se trasluce a través de los comentarios de Katori e Itasaka. Como hemos señalado, Mishima anhela aprender a bailarlo, así como escribir en japonés obras teatrales de inspiración flamenca donde el baile y el cante sean su eje articulador. Tras esto, el encuentro con Díez del Corral dota al escritor de una serie de conocimientos teóricos que le permiten comprender los mecanismos histórico-culturales que subyacen no solo en el flamenco, sino también en el mundo del toro y en la cultura española en general, haciendo que un año antes de su fatal suicidio, escribiese sobre el "alma española de los samuráis".

Una segunda conclusión es la siguiente: a ojos de Mishima, España es considerada geográficamente europea, pero culturalmente ajena a la órbita puritana occidental. España representa la especificidad en la unidad, o lo que es lo mismo, la pieza del puzle que no acaba de encajar en el conjunto general europeo u occidental. El hecho de que España no sea considerada como territorio europeo por parte de los países del entorno aparece sugerido ya en el primer texto que hemos presentado. En este caso Mishima habla de oídas y enfatiza que dicha consideración despectiva es "lo que se dice”. Sin embargo, con el pasar de los años, esta especificidad española se vuelve más clara a sus ojos, lo cual hace que llegue al punto de asimilar España a Japón y coloque a ésta en una posición claramente antagónica a los demás países occidentales. ¿Quiere Mishima decir con eso que España no forma parte de Occidente? Y en caso de que no forme parte, ¿quiere eso decir que forma un bloque cultural autónomo e independiente del entorno? Son estas unas cuestiones bastante difíciles de dirimir teniendo en cuenta los escasos textos existentes. Lo que sí resulta manifiesto es que para Mishima, sobre todo en sus últimos años, la cuestión de "lo español" aparece siempre ligada a "lo japonés". Es decir, no trata el tema de España de forma autónoma, sino que en cada ocasión lo presenta como término de comparación, como ejemplo a seguir para los japoneses a nivel cultural. En opinión de Mishima, España aparece como punto de referencia, como ejemplo de país que, pese a la presión exterior, conservó su base histórico-cultural y rechazó adoptar unos modos extranjeros aparentemente universales que le resultan ajenos y que, en última instancia, van en contra de su esencia.

Una tercera conclusión, y que está relacionada con la anterior, es que en España, a diferencia de Japón, existe una válvula de escape a la presión cultural propia y extranjera representada por el flamenco, pero, sobre todo, por la tauromaquia. Esta válvula de escape tiene, a través de los sentimientos exacerbados, la violencia y el derramamiento de sangre, un efecto catártico sobre los individuos de la sociedad. De ahí que Mishima utilice la analogía del volcán en numerosas ocasiones: Japón, culturalmente oprimido por el peso de la cultura occidental, a través de sus grietas varias (como son los casos mencionados del asesinato del líder del Partido Comunista Asanuma Inejirō, el ataque al Secretario de Estado Americano William Rogers o incluso el suicidio que el propio Mishima lleva a cabo) deja escapar lava de cuando en vez. Ello se debe a que la apariencia de éxito económico y tecnológico que proyecta el país es meramente superficial. El estrés cultural de intentar ser alguien diferente o de adoptar una tradición cultural ajena y tan alejada de las coordenadas culturales propias podría producir, tarde o temprano, una fatal erupción. En este sentido, la semejanza entre la cultura española y la japonesa en términos de escala de valores y presencia de elementos escatológicos, se torna en una gran diferencia entre ambos países ante el hecho de aceptar o no la propia especificidad.

Una última conclusión que se puede sacar de los textos presentados es la relativa al concepto de tauroflamencología que da título al presente trabajo. Es de todos conocido que la imagen exterior de España tiene su eje articulador en las artes del flamenco y la tauromaquia, por lo que el hecho de que un joven Mishima presente nuestro país a través de descripciones costumbristas de volantes, mantillas y ojos negros de largas pestañas no puede considerarse demasiado innovador. Lo que sí resulta llamativo en el caso de Mishima es que, tras haber cimentado una sólida base de conocimientos sobre nuestro país, viese la conexión que subyace al mundo del flamenco y el toro al atribuirle a ambos una labor catárquica similar. El concepto de tauroflamencología que mencionamos al comienzo del presente trabajo es muy reciente, de los años 90 del siglo pasado, y los estudios existentes hasta el momento son muy escasos. Que el propio Mishima fuese capaz de conectar la función de ambas artes y encontrar puntos de unión entre ellas, posiblemente de forma intuitiva, resulta cuanto menos sorprendente teniendo en cuenta el escasísimo número de estudios científicos que existían en la época.

\section{Bibliografía}

Arriazu, Santy (2006): Antonio el Bailarín: memorias de viva voz. Barcelona: Ediciones B.

Díez del Corral, Luis (1954): El rapto de Europa. Una interpretación histórica de nuestro tiempo. Madrid: Revista de Occidente.

Escudero Urive, Vicente (2017): Mi baile: seguido de Pintura que baila; Decálogo del baile flamenco; El enigma de Berruguete; Arte flamenco jondo. Sevilla: Athenaica. 
González, Juan Antonio (2013): España y Europa en el pensamiento de Luis Díez del Corral. Huelva: Servicio de Publicaciones de la Universidad de Huelva.

Gutiérrez-Solana, José (ed. Andrés Trapiello) (2000): La España negra [1920]. Granada: Comares.

Ibáñez, Alberto (2018): La Leyenda Negra: Historia del odio a España. Córdoba: Almuzara.

Itasaka, Gō (1997): Kyokusetsu Mishima Yukio. Seppuku to furamenko. Tōkyō: Natsume Shobō.

Katori, Kiyoko (1998): Hachijū go sai, shinayakani furamenko. Tōkyō: Paseo.

Kojima, Takehiko (1969): Kako to Mirai. Tōkyō: Miraisha.

López Rodríguez, Fernando (2007): De puertas para adentro. Disidencia sexual y disconformidad de género en la tradición flamenca. Madrid: Egales.

Mishima, Yukio (1958): Tabi no ehon. Tōkyō: Kōdansha.

Mishima, Yukio (1969): A problem of culture. The Times, 24 de septiembre.

Mishima, Yukio (1969): Bunka Bōeiron. Tōkyō: Shinchōsha.

Mishima, Yukio (1975): Zenshū. Tōkyō: Shinchōsha.

Mishima, Yukio (trad. Carlos Rubio) (2015): Últimas palabras de Yukio Mishima. Madrid: Alianza Editorial.

Noya, Javier (2012): La imagen de España en el mundo. Madrid: Tecnos.

Regoyos, Darío de / Verhaeren, Emile (1899): España negra. Barcelona: Imprenta de Pedro Ortega.

Ríos Ruíz, Manuel (2019): Ayer y Hoy del cante flamenco. Madrid: Istmo.

Shinoyama Kishin (2000): Mishima Yukio no ie. Tōkyō: Bijutsu Shuppansha.

Trapiello, Andrés (2000): "Prólogo". En: Gutiérrez-Solana, José. La España negra [1920]. Granada: Comares.

Vallejo-Nájera, Juan Antonio (1978): Mishima o el placer de morir. Barcelona: Planeta, 1978. 